\title{
Overexpression of mitochondrial Hsp75 protects neural stem cells against microglia-derived soluble factor-induced neurotoxicity by regulating mitochondrial permeability transition pore opening in vitro
}

\author{
YAN WANG $^{1,2^{*}}$, JIZONG LIN ${ }^{3 *}$, QING-ZHUANG CHEN ${ }^{1}$, NING ZHU ${ }^{1}$, DE-QI JIANG ${ }^{1}$, \\ MING-XING LI ${ }^{1}$ and YONG WANG ${ }^{1}$ \\ ${ }^{1}$ Department of Pharmacy, Zhujiang Hospital, Southern Medical University, Guangzhou, Guangdong 510282; \\ ${ }^{2}$ Department of Pharmacy, Guangdong Hospital of Integrated Chinese and Western Medicine, Foshan, Guangdong 528200; \\ ${ }^{3}$ Department of Hepatobiliary Surgery, The Third Affiliated Hospital of Sun Yat-Sen University, \\ Guangzhou, Guangdong 510630, P.R. China
}

Received May 21, 2015; Accepted September 17, 2015

DOI: $10.3892 /$ ijmm.2015.2380

\begin{abstract}
Microglia (MG)-induced neurotoxicity, a major determinant of Alzheimer's disease, is closely related to the survival of neural stem cells (NSCs). Heat shock protein 75 (Hsp75) has been reported to exert protective effects against environmental stresses; however, whether or not it protects NSCs against MG-derived soluble factor-induced neurotoxicity remains unclear. In the present study, we constructed NSCs that overexpressed human Hsp75 protein and established a co-culture system in order to elucidate the role of Hsp75 in NSC-MG interactions. The results obtained indicated that Hsp75 expression increased after $12 \mathrm{~h}$ of soluble factor induction and continued to increase for up to $36 \mathrm{~h}$ of treatment. The overexpression of Hsp75 decreased NSC apoptosis and preserved mitochondrial membrane potential. Further experiments revealed that the overexpression of Hsp75 inhibited the formation of cyclophilin $\mathrm{D}(\mathrm{CypD})$-dependent mitochondrial permeability transition pore (mPTP) involvement in neurotoxicity-mediated mitochondrial dysfunction and suppressed the activation of the mitochondrial apoptotic cascade, as demonstrated by the inhibition of the release of cytochrome $c$ (Cytc) and the activation of caspase-3. The findings of this study demonstrate that Hsp75 overexpression prevents the impairment of NSCs induced by MG-derived soluble factors by regulating the opening of mPTP. Thus,
\end{abstract}

Correspondence to: Professor Yong Wang, Department of Pharmacy, Zhujiang Hospital, Southern Medical University, 253 Industrial Road, Haizhu, Guangzhou, Guangdong 510282, P.R. China

E-mail: yongwh2005@163.com

${ }^{*}$ Contributed equally

Key words: heat shock protein 75, microglia-induced neurotoxicity, Alzheimer's disease, neural stem cells
Hsp75 warrants further investigation as a potential candidate for protection against neurotoxicity.

\section{Introduction}

Alzheimer's disease (AD) is a chronic neurodegenerative disease that is characterized by progressive learning and memory loss, the incidences of which increase with age. The classical pathological hallmarks of $\mathrm{AD}$ are an intracellular accumulation of amyloid plaques and neurofibrillary tangles (NFTs) along with the loss of neurons $(1,2)$. The $\beta$-amyloid peptide $(A \beta)$ is the main component of amyloid plaques, and it plays a vital role in the formation of both amyloid plaques and NFTs. In addition, $A \beta$ interactions with receptors (e.g., ryanodine receptors, the $\mathrm{N}$-methyl-D-aspartate receptor, $\mathrm{N}$-formyl peptide receptor like-1, CD36 receptor and $\alpha 7$ nicotinic acetylcholine receptor) which are expressed in brain cells [e.g., neuronal cells, endothelial cells and microglia (MG)/microglial (MG) cells] induce neurotoxicity (3). MG cells are the resident antigen-presenting cells in the central nervous system (CNS). They can be activated in response to environmental toxins and are known to display diverse reactions that are associated with both protective and deleterious effects. It is widely accepted that $\mathrm{A} \beta$ can accelerate neurodegeneration by activating MG cells $(4,5)$ and that MG cell activation results in the release of soluble factors, including reactive oxygen species, various inflammatory mediators and chemokines. When neurons are exposed to an excess of soluble factors, neuronal apoptosis increases. Although neural stem cells (NSCs) are capable of self-renewal and are multipotent cells that can differentiate into the main cell phenotypes of the CNS in order to maintain CNS homeostasis $(6,7)$, the proliferation and differentiation of NSCs are also suppressed due to the release of numerous soluble factors. Therefore, one possible treatment strategy for AD would be to protect NSCs against MG-derived soluble factors. The murine MG cell line, BV-2, has been used previously as a substitute for primary MG cells, as it exhibits very similar behavior (8). 
In addition, the C17.2 NSC line is capable of self-renewal and differentiation (9) and has been used as a model system for neurodegenerative diseases (10). Thus, in the present study, we used BV-2 cells and C17.2 cells as replacements for primary MG cells and primary NSCs, respectively.

Previous studies have confirmed that the mitochondrial permeability transition pore (mPTP) plays an important role in cell apoptosis. The opening of the mPTP results in potential dissipation of the membrane, mitochondrial swelling, rupture of the outer membrane, the release of pro-apoptotic proteins, such as cytochrome $c$ (Cytc) and the induction of caspase-3like activity, which eventually initiates apoptosis (11-13). Indeed, irreversible mitochondrial impairment caused by mPTP opening is a key step in apoptosis under A $\beta$-induced neurotoxic, and other, conditions. These findings suggest that mPTP activation is a key player in cell death and is a potential target for cytoprotective intervention.

Heat shock protein 75 (Hsp75) is a member of the Hsp70 chaperone family and is expressed predominantly in mitochondria (14) as a marker of stress. Hsp75 is not heat-inducible, but it has been reported to respond to other forms of stress, including glucose deprivation, oxidative injury, focal ischemia and certain drugs (15-20). However, it remains unclear as to whether Hsp75 can respond to MG-derived soluble factors. Thus, in this study, we examined Hsp75 expression in NSCs in response to MG-derived soluble factors. Recent studies have revealed that Hsp75 expression plays a vital role in maintaining mitochondrial function and cell survival under various pathological conditions, including AD (21-23). However, further details of the neuroprotective mechanisms associated with Hsp75 remain to be elucidated, particularly its anti-apoptotic effects on NSCs. We hypothesized that the overexpression of Hsp75 would inhibit the formation of cyclophilin D (CypD)dependent mPTP opening and reduce the release of Cytc into the cytosol following treatment with $\mathrm{A} \beta_{1-42}$ in an NSC-MG cell co-culture system. To examine this hypothesis, NSCs overexpressing Hsp75 protein were constructed and then subjected to the above-mentioned treatment. Apoptosis was evaluated by flow cytometry. In addition, changes in the protein expression of related proteins were assessed by western blot analysis.

The purpose of this study was to investigate changes in Hsp75 expression following treatment with soluble factors and to observe whether Hsp75 overexpression provides protection against MG-derived soluble factor-induced neurotoxicity by regulating $\mathrm{mPTP}$ opening.

\section{Materials and methods}

Cell culture and the NSC-MG cell co-culture system. In the present study, we used BV-2 cells and C17.2 cells as replacements for primary MG cells and primary NSCs, respectively. The immortalized murine NSC line, $\mathrm{C} 17.2$, was generously provided by Professor Wei Lin Jin (Shanghai Jiao Tong University, Shanghai, China). The C17.2 cells were cultured in high glucose Dulbecco's modified Eagle's medium (DMEM) containing $10 \%$ fetal bovine serum (FBS), $5 \%$ horse serum, and $2 \mathrm{mM}$ glutamine. The murine MG cell line, BV-2, was a gift from Professor Ai Min Ji (Southern Medical University, Guangzhou, China). The BV-2 cells were propagated in flasks containing DMEM supplemented with $10 \% \mathrm{FBS}$, at $37^{\circ} \mathrm{C}$ with $5 \% \mathrm{CO}_{2}$. Cells in the exponential growth phase were used for the experiments. The morphology of C17.2 cells was observed under a microscope.

The MG cells were cultured in a Transwell system (3450; Corning Corp., Corning, NY, USA) that was placed above the NSC layer. The NSCs and MG cells shared the same medium, but had no direct cell-cell interactions, as the cells were physically separated with a polyester membrane. The pore size of the Transwell $(0.4 \mu \mathrm{m})$ does not permit cell migration through the membrane. The MG cells were then stimulated with $\mathrm{A} \beta_{1-42}$ $(10 \mu \mathrm{M})$. In the presence of inserts containing MG cells, on top of the NSC layer, we observed the response of the NSCs to the diffusible factors secreted by the stimulated MG cells. C17.2 cells in the control group were placed in the lower chamber and the upper chamber was left empty. C17.2 cells were placed in the lower chamber in a final concentration of $10 \mu \mathrm{M} \mathrm{A} \beta_{1-42}$ (group of C17.2 cells directly exposed to $\mathrm{A} \beta_{1-42}$ ).

Preparation of $A \beta_{1-42}$. A $\beta_{1-42}$ (US Biological, Salem, MA, USA) was dissolved in $35 \%$ acetonitrile and then further diluted to $10 \mathrm{mM}$ with phosphate-buffered saline (PBS). The peptide solution was subsequently incubated at $37^{\circ} \mathrm{C}$ for $72 \mathrm{~h}$ to promote aggregation and fibrillization, followed by freezing and storage at $-20^{\circ} \mathrm{C}$. The final working concentration was $10 \mu \mathrm{M} \mathrm{A} \beta_{1-42}$ diluted in culture medium.

Recombinant adenoviral vector for Hsp75 overexpression. A recombinant adenoviral vector overexpressing Hsp75 (Ad-Hsp75-GFP) and a negative control adenoviral vector (Ad-GFP) were produced by HanBio (Shanghai, China). The vectors encoded the green fluorescent protein (GFP) sequence, which served as a marker gene. When the $\mathrm{C} 17.2$ cells reached $60 \%$ confluence, recombinant adenovirus was added at a multiplicity of infection of 100 for $36 \mathrm{~h}$. The cells were observed under a fluorescence microscope (Olympus, Tokyo, Japan) to detect the presence of fluorescent protein. All the recombinant adenoviruses were tested for Hsp75 protein expression in the C17.2 cells by western blot analysis.

Isolation of mitochondria. Mitochondria were isolated from the NSCs (C17.2 cells) using the Mitochondria Isolation kit for cultured cells (Thermo Fisher Scientific, Waltham, MA, USA) according to the manufacturer's instructions. Briefly, the samples were harvested, and this was followed by the addition of $800 \mu \mathrm{l}$ mitochondria isolation reagent $\mathrm{A}, 10 \mu \mathrm{l}$ mitochondria isolation reagent $\mathrm{B}$ and $500 \mu \mathrm{l}$ mitochondrial isolation reagent $\mathrm{C}$ (from Mitochondria Isolation kit for cultured cells; Thermo Fisher Scientific, Waltham, MA, USA). Cytosolic and mitochondrial proteins were harvested according to the manufacturer's instructions and then concentrated using an Amicon ${ }^{\circledR}$ Ultra-0.5 Centrifugal Filter device (Millipore, Billerica, MA, USA).

Cell viability assays. The apoptotic rates of the NSCs (C17.2 cells) were examined using an Annexin V-APC/7-AAD apoptosis detection kit (BD Pharmingen, San Diego, CA, USA) in accordance with the manufacturer's instructions. Following treatment with $A \beta_{1-42}$ for $36 \mathrm{~h}$, the cells were harvested and resuspended in binding buffer at a density of $1 \times 10^{6}$ cells $/ \mathrm{ml}$. The cells were mixed with $5 \mu \mathrm{l}$ Annexin V-APC and $5 \mu \mathrm{l}$ 7-AAD and then incubated for $15 \mathrm{~min}$ at room temperature in the dark. Finally, the cells were analyzed using a flow cytometer (BD Pharmingen). 
Western blot analysis and reverse transcription-quantitative polymerase chain reaction $(R T-q P C R)$. Following treatment, the $\mathrm{C} 17.2$ cells were collected and lysed for protein expression analysis. The protein concentration was determined using the BCA protein assay kit (Thermo Fisher Scientific) with bovine serum albumin as a standard. Total protein equivalents were denatured and separated on a $10-15 \%$ sodium dodecyl sulfate (SDS)-polyacrylamide gel, and the proteins were then transferred onto Immobilon-P Transfer membranes (Millipore). The membranes were blocked with 5\% non-fat dry milk for 1 hat room temperature and then incubated with primary antibodies. The primary antibodies were as follows: Hsp75 (ab151239; Abcam, Cambridge, MA, USA), CypD (A3208; ABclonal, Cambridge, MA, USA), Cytc (ab133504; Abcam), caspase-3 (WL0146; Wanleibio, Shenyang, China), COX IV (internal control for mitochondrial protein; ab140643; Abcam), glyceraldehyde 3-phosphate dehydrogenase (GAPDH; KC-5G5) and $\beta$-actin (KC-5A08; both from KangChen Bio-tech, Shanghai, China) and $\alpha$-tubulin (AT819; Beyotime Institute of Biotechnology, Haimen, China). The secondary antibodies were either goat anti-rabbit (BA1054; Boster Biotech Co., Ltd., Wuhan, China) or mouse (bs-0296G-HRP; Bioss Co., Beijing, China) immunoglobulin $\mathrm{G}$ ( $\mathrm{IgG})$. Horseradish-conjugated secondary antibody labeling was detected using enhanced chemiluminescence (Thermo Fisher Scientific) according to the manufacturer's instructions. The optical density of the bands was analyzed with ImageJ software.

For RT-qPCR, total RNA was extracted from the C17.2 cells using TRIzol ${ }^{\circledR}$ reagent (Invitrogen, Carlsbad, CA, USA). The RNA $(2 \mu \mathrm{g})$ was reverse transcribed into cDNA using ReverTra Ace $^{\circledR}$ qPCR RT Master Mix with the gDNA Remover kit (Toyobo, Osaka, Japan). Real-time monitoring of the PCR amplification of cDNA was detected using a real-time PCR detection system (ABI PRISM ${ }^{\circledR} 7500$ sequence detection system). PCR amplification was conducted as follows: 40 cycles at $95^{\circ} \mathrm{C}$ for $15 \mathrm{sec}$ and an extension at $60^{\circ} \mathrm{C}$ for $32 \mathrm{sec}$. The data were quantified using the $2^{-\Delta \Delta \mathrm{Ct}}$ method. The following primers were used: mouse CypD, 5'-AACTTCAGAGCCCTATGCA-3' (forward) and 5'-TCCTGTGCCATTGTGGTT-3' (reverse); mouse caspase-3, 5'-GTTCATCCAGTCCCTTTGC-3' (forward) and 5'-TGTTAACGCGAGTGAGAATG-3' (reverse); mouse $\beta$-actin, 5 '-GCTTCTAGGCGGACTGTTAC-3' (forward) and 5'-CCATGCCAATGTTGTCTCTT-3' (reverse). $\beta$-actin (a housekeeping gene) was used as an internal reference.

Immunocytochemistry. Cell cultures in 96-well plates were examined by fluorescence immunocytochemistry. The cells were washed twice in PBS and then fixed in 4\% paraformaldehyde for $30 \mathrm{~min}$ at room temperature. The cells were then washed twice with PBS and permeabilized with $0.3 \%$ Triton X-100 for $20 \mathrm{~min}$. Non-specific binding sites were blocked via incubation with $3 \%$ goat serum for $40 \mathrm{~min}$. The cells were then incubated with a mouse monoclonal primary antibody against nestin (a marker of NSCs) (ab6142; Abcam) at a dilution of 1:200 in 1\% BSA overnight at $4^{\circ} \mathrm{C}$. On the following day, the cells were washed 3 times for 5 min each time and incubated with rhodamine-conjugated goat anti-mouse secondary antibody (SA00006-1; Proteintech, Chicago, IL, USA) at room temperature for $1.5 \mathrm{~h}$ in the dark; this was followed by 2 washes in PBS. Subsequently, the nuclei were counterstained with 4',6-diamidino-2-phenylindole (DAPI), and the stained cells were observed under a fluorescence microscope (Olympus AX80; Olympus).

Mitochondrial membrane potential. The mitochondrial membrane potential was monitored using the fluorescent dye, tetramethylrhodamine ethyl ester (TMRE; Molecular Probes, Eugene, OR, USA). Following treatment, the cells were washed with PBS and incubated in the dark with $100 \mathrm{nM}$ TMRE at $37^{\circ} \mathrm{C}$ for $15 \mathrm{~min}$. The cells were then washed 3 times with PBS and observed under a fluorescence microscope (Olympus AX80; Olympus).

Statistical analysis. Data are expressed as the means \pm standard error of the mean (SEM). Differences among groups were analyzed by one-way ANOVA followed by the least significant difference (LSD) or Dunnett's T3 post-hoc test. A p-value $<0.05$ was considered to indicate a statistically significant difference.

\section{Results}

The stimulation of $M G$ cells with $A \beta_{1-42}$ increases Hsp75 expression in $C 17.2$ cells. Western blot analysis was used to measured the Hsp75 expression levels following treatment with $A \beta_{1-42}$ in the NSC-MG co-culture system. The level of Hsp75 increased in the $\mathrm{C} 17.2$ cells following direct exposure to $\mathrm{A} \beta_{1-42}$ (+A $\beta$ group). Although Hsp75 expression was low in the control group, the levels of Hsp75 in the C17.2 cells gradually increased from 12 to $36 \mathrm{~h}$ following treatment of the MG cells with $\mathrm{A} \beta_{1-42}$ in the co-culture system (Fig. 1). Based on this result, $36 \mathrm{~h}$ was used as the optimal treatment time in subsequent experiments.

Verification of Ad-GFP and Ad-Hsp75 transfection. We constructed a recombinant adenoviral vector for transfection using a GFP tag. Following $36 \mathrm{~h}$ of infection, the infection efficiency was visualized by measuring GFP expression (Fig. 2A). At $36 \mathrm{~h}$ following transfection, C17.2 cells were shuttle-like or irregular shaped and no obvious changes in the morphology of the C17.2 cells were observed among these groups (Fig. 2B). In addition, we detected the expression of nestin (a NSC marker) in 3 groups (control group, Ad-GFP group and Ad-Hsp75 group). As shown in Fig. 2C, C17.2 NSCs were all nestin-positive. Additionally, Hsp75 expression increased significantly in the C17.2 cells that were infected with Ad-Hsp75 compared to those that were infected with the negative control vector (Ad-GFP), and to the endogenous Hsp75 levels in the control cells, as indicated by the results of the western blot analysis (Fig. 3).

Hsp75 overexpression protects C17.2 cells against soluble factor-induced neurotoxicity. As Hsp75 expression in the NSCs increased following treatment with soluble factors $\left(\mathrm{A} \beta_{1-42}\right)$, we thus performed experiments to determine whether this increase in Hsp75 expression exerts a protective effect against the neurotoxicity induced by diffusible soluble factors. To investigate the effects of Hsp75 overexpression on neurotoxicity, a C17.2 cell line overexpressing human Hsp75 was established. We then investigated the effects of Hsp75 overexpression on soluble factor-induced apoptosis in the C17.2 cells. Apoptotic-like cell death was assayed by flow cytometry using Annexin V-APC and 7-AAD (Fig. 4). Following treatment of the C17.2 cells with $\mathrm{A} \beta_{1-42}$, the percentage of apoptotic cells increased to 
A

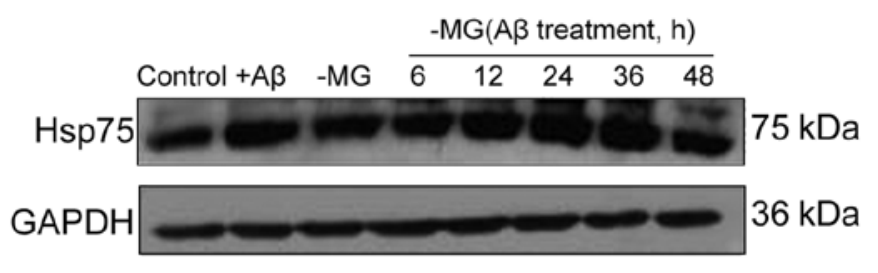

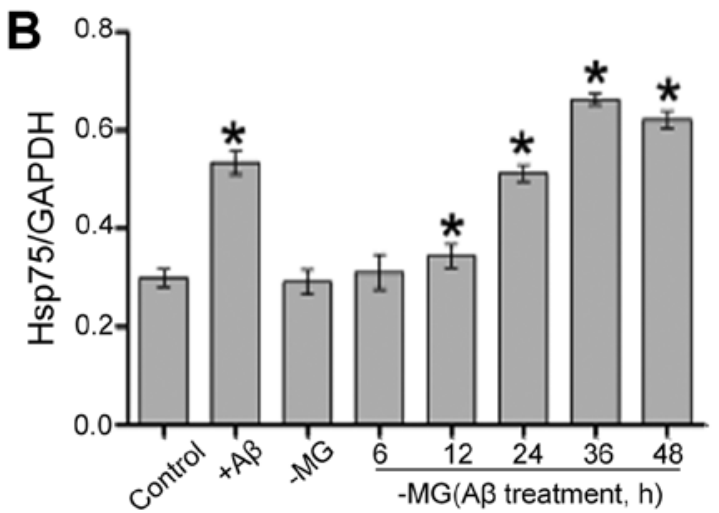

Figure 1. Effects of microglial (MG)-derived soluble factors on the level of heat shock protein 75 (Hsp75) in neural stem cells (NSCs). (A) Western blot analysis showing Hsp75 levels in the co-culture system. (B) Hsp75 levels were normalized to GAPDH in the co-culture system. Data are the means \pm SEM. "p $<0.05$ compared to the control group. The experiment was repeated 3 times. A $\beta, \beta$-amyloid peptide.

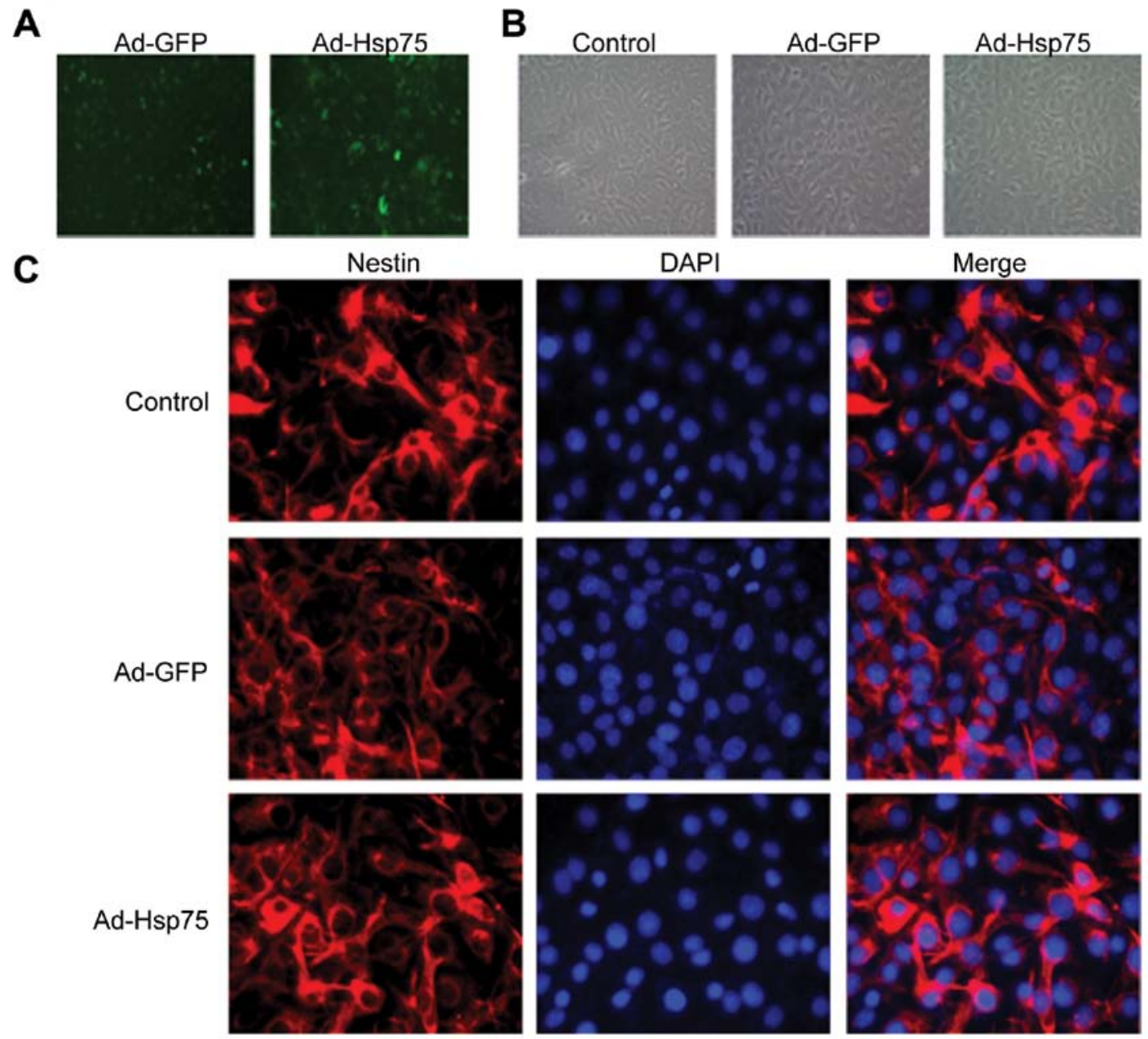

Figure 2. Photomicrographs showing C17.2 cells transfected with adenovirus. (A) C17.2 cells were infected with adenovirus green fluorescent protein (Ad-GFP) or adenovirus heat shock protein 75 (Ad-Hsp75) for $36 \mathrm{~h}$ and observed under a fluorescence microscope to determine the infection efficiency by visualizing the expression of GFP. Magnification, x100. (B) Representative phase contrast micrographs showing the morphology of C17.2 cells. C17.2 cells were transfected with Ad-GFP or Ad-Hsp75 for $36 \mathrm{~h}$. The morphology of C17.2 cells was observed using a phase contrast microscope. Magnification, x200. (C) Detection of nestin protein expression in C17.2 cells following infection. Magnification, $\mathrm{x} 400$.

approximately $12 \%$ compared with only $4 \%$ in the control group $(\mathrm{p}<0.05)$, which demonstrated that $\mathrm{A} \beta_{1-42}$ at a concentration of $10 \mu \mathrm{M}$ induced cell apoptosis. In the co-culture system, the proportion of apoptotic NSCs was approximately $4 \%$ in the NSC-MG cell co-culture system without $\mathrm{A} \beta_{1-42}$ treatment (-MG group), and there was no apparent difference $(p>0.05)$ between the cells in the NSC-MG co-culture system without treatment with $\mathrm{A} \beta_{1-42}$ and the control group. 
A

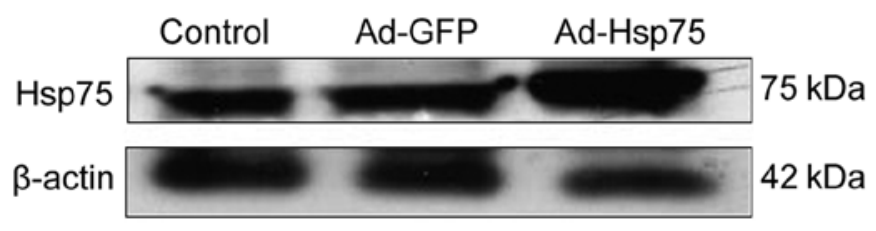

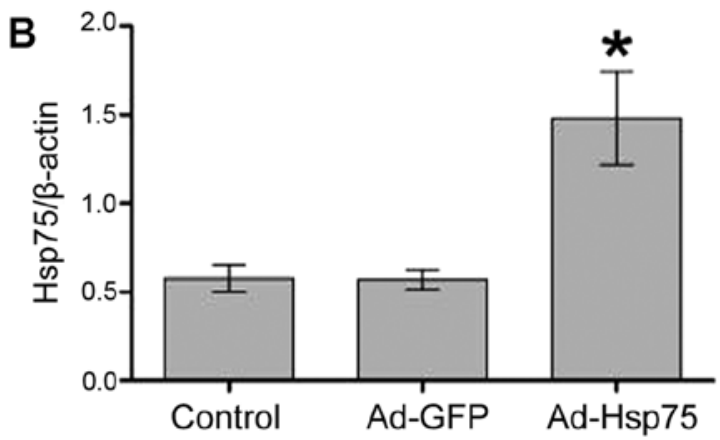

Figure 3. Hsp75 expression levels following infection. (A) Western blot analysis of Hsp75 protein expression in the uninfected control (endogenous) and in adenovirus green fluorescent protein (Ad-GFP)-infected and adenovirus heat shock protein 75 (Ad-Hsp75)-infected C17.2 cells. (B) Hsp75 levels were normalized with $\beta$-actin. ${ }^{*} \mathrm{p}<0.05$ compared with the control group and Ad-GFP group. Data represent the means \pm SEM. Representative results of 3 independent experiments are shown.

A

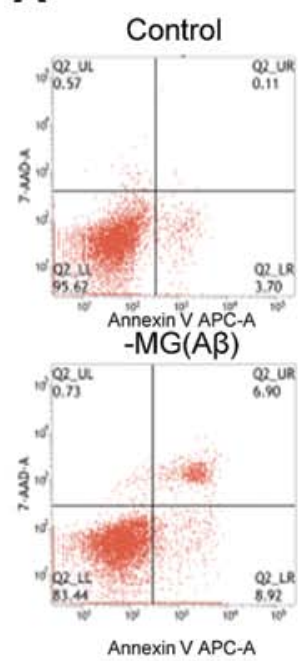

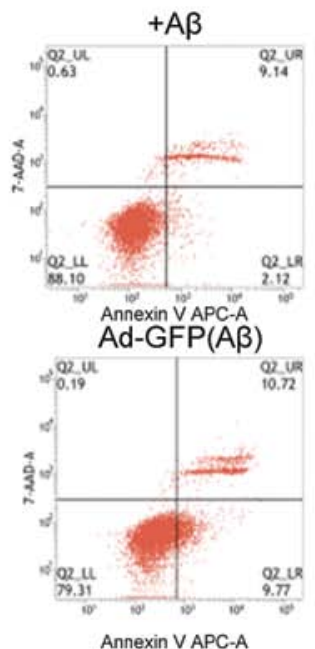

Annexin V APC-A

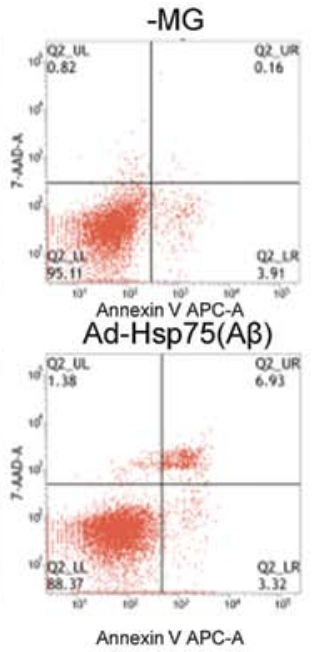

B

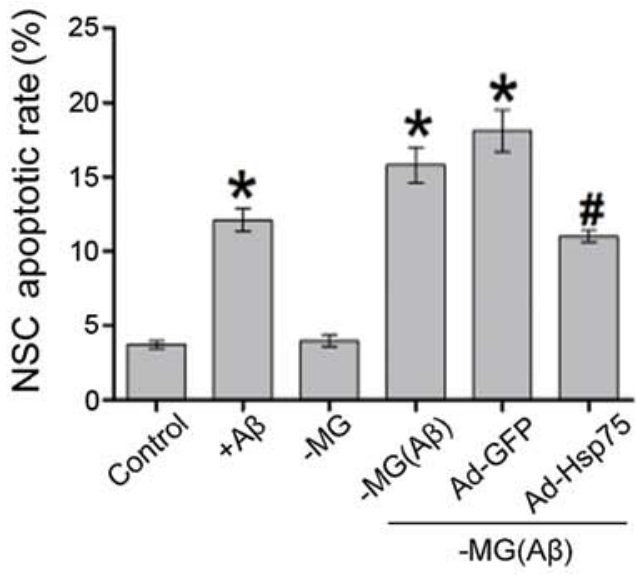

Figure 4. Effect of Hsp75 overexpression on soluble factor-induced apoptosis. (A) Neural stem cells (NSCs) were double-stained with Annexin V-APC and 7-AAD and then analyzed by flow cytometry. (B) Representative histogram of the NSC apoptotic rate in different groups. Early and late apoptotic cell proportionslevels were calculated. Data represent the means \pm SEM. ${ }^{*} \mathrm{p}<0.05$ compared to the control group; ${ }^{*} \mathrm{p}<0.05$ compared to the NSC-microglial (NSC-MG) cells treated with $\beta$-amyloid peptide $(\mathrm{A} \beta)_{1-42}$ and the adenovirus green fluorescent protein (Ad-GFP) group. Three independent experiments were performed.

When the MG cells were treated with $A \beta_{1-42}$ for $36 \mathrm{~h}$ in the co-culture system, the percentage of early and late apoptotic cells markedly increased, and also increased in the Ad-GFP group, compared with the control group (p<0.05) (Fig. 4B). By contrast, Hsp75 overexpression markedly prevented MG-derived soluble factor-induced apoptosis. These results indicate that Hsp75 overexpression provides protection against MG-derived soluble factor-induced neurotoxicity in NSCs.

Hsp75 overexpression attenuates soluble factor-induced mitochondrial dysfunction. As mitochondrial membrane potential is a key indicator of cell apoptosis, we speculated that soluble factor-induced neurotoxicity correlates with mitochondrial dysfunction. To examine the effects of Hsp75 overexpression on mitochondrial dysfunction, mitochondrial membrane potential was monitored using the fluorescent dye, TMRE. As shown in Fig. 5, compared with the control group, mitochondrial membrane potential significantly decreased in the $C 17.2$ cells that were exposed directly to $A \beta_{1-42}$. However, there was no apparent difference between the NSC-MG cell group (in the co-culture system) without treatment with $\mathrm{A} \beta_{1-42}$ and the control group. Additionally, in the co-culture system, mitochondrial membrane potential in the presence of Hsp75 overexpression was protected against diffusible soluble factormediated neurotoxicity. By contrast, the TMRE signal was reduced in the NSC-MG cells treated with $\mathrm{A} \beta_{1-42}$ and in the adenoviral vector-infected C17.2 cells (Ad-GFP group). The above-mentioned results indicate that Hsp75 overexpression can effectively prevent soluble factor-induced mitochondrial dysfunction.

Hsp75 overexpression reduces soluble factor-induced CypDdependent mPTP opening. Previous studies have confirmed that mitochondrial membrane potential dissipation is accompanied by an increase in MPTP opening and indirectly reflects the state of $\mathrm{mPTP}$ opening (24-26). Additionally, it has been demonstrated that CypD is an important regulatory component of $\mathrm{mPTP}$ and plays a vital role in regulating mPTP opening (27-29). Increasing evidence suggests that an increased abundance of CypD directly reflects the state of mPTP opening and that CypD-mediated mPTP opening is closely related to cell viability (30-32). Thus, in order to deter- 


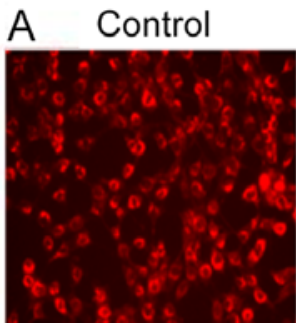

$-M G(A \beta)$

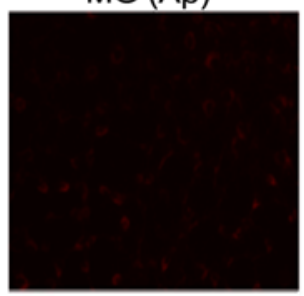

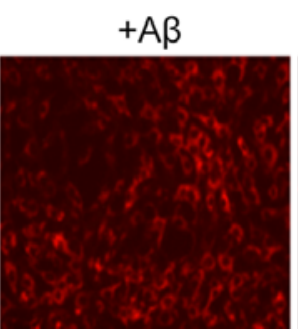

$\operatorname{Ad-GFP}(A \beta)$

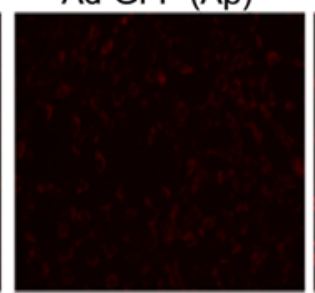

$-M G$

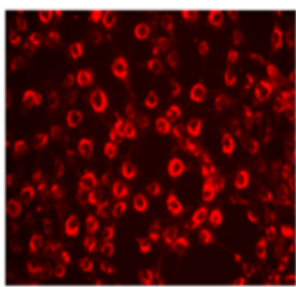

$\operatorname{Ad}-\mathrm{Hsp} 75(\mathrm{~A} \beta)$

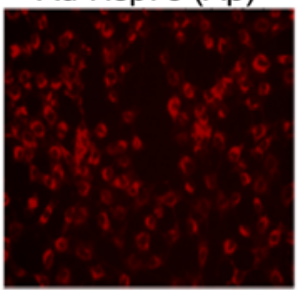

B

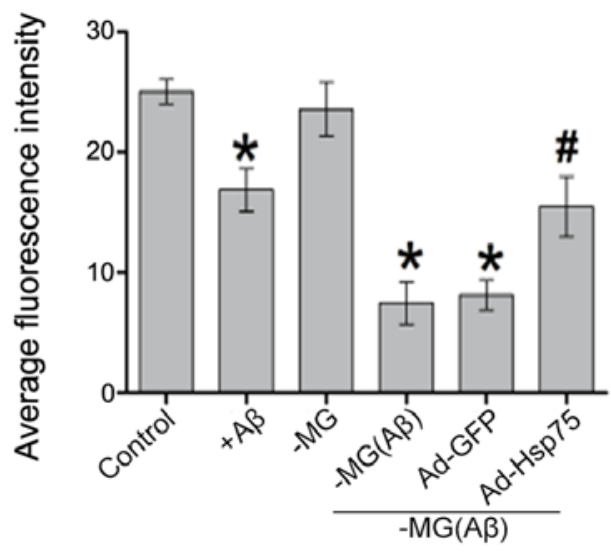

Figure 5. Detection of mitochondrial membrane potential. (A) Following treatment, the NSCs were stained with TMRE and detected using a fluorescence microscope. Magnification, x200. (B) Average fluorescence intensity. ${ }^{*} \mathrm{p}<0.05$ compared to the control group; ${ }^{*} \mathrm{p}<0.05$ compared to the neural stem cell-microglial (NSC-MG) cells treated with $\beta$-amyloid peptide $(\mathrm{A} \beta)_{1-42}$ and the adenovirus green fluorescent protein (Ad-GFP) group. Ad-Hsp, adenovirus heat shock protein.

A

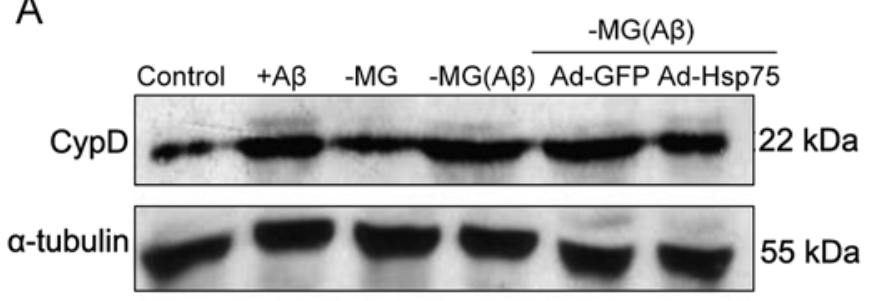

B
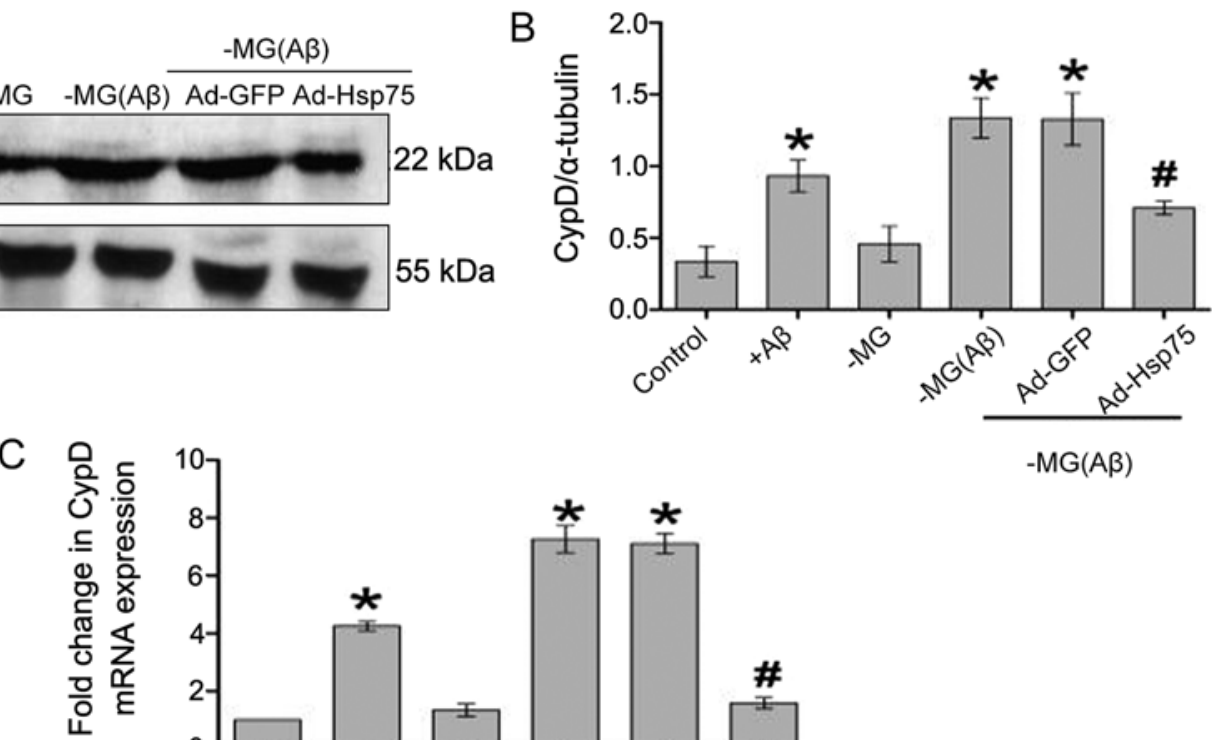

$-M G(A \beta)$

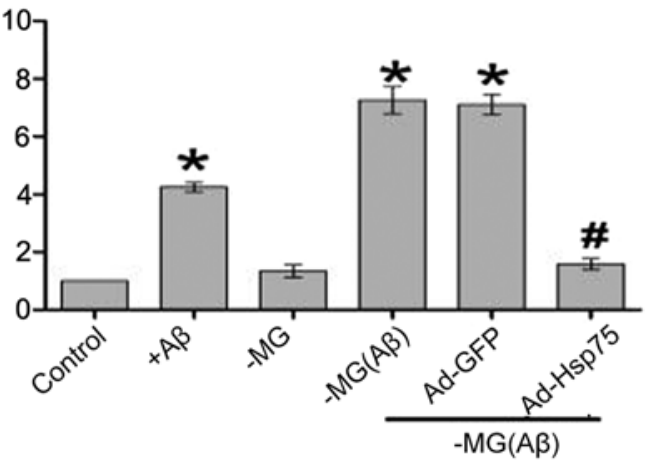

Figure 6. The expression of cyclophilin D (CypD) was analyzed by western blot analysis and RT-qPCR. (A) Western blot analysis of CypD and $\alpha$-tubulin protein expression in NSCs following treatment. (B) Quantitative analysis of CypD protein expression. The expression of the targeted protein was determined relative to the expression of $\alpha$-tubulin protein. (C) RT-qPCR analysis of CypD mRNA levels expressed in NSCs following treatment. The RT-qPCR experiment was done performed in triplicate, and CypD mRNA expression was normalized to the expression of $\beta$-actin. Data represent the means \pm SEM. "p $<0.05$ compared to the control group; ${ }^{*} \mathrm{p}<0.05$ compared to the neural stem cell-microglial (NSC-MG) cells treated with $\beta$-amyloid peptide (A $\left.\beta\right)_{1-42}$ and the adenovirus green fluorescent protein (Ad-GFP) group. Three independent experiments were performed. Ad-Hsp75, adenovirus heat shock protein 75.

mine whether Hsp75 overexpression protects NSCs against MG-derived soluble factor-induced neurotoxicity by regulating mPTP opening, we estimated the relative abundance of CypD (Fig. 6). Briefly, compared with the control group, the CypD expression levels were upregulated in the C17.2 cells following direct exposure to $A \beta_{1-42}$, which is consistent with the results of a previous study (33). According to the results of western blot analysis, the CypD levels in the soluble factor-stimulated group in our co-culture system (NSC-MG cell group with $A \beta_{1-42}$ treatment and the Ad-GFP group) were significantly increased as compared to the non-soluble factor group (control group). By contrast, the CypD levels were downregulated in response to Hsp75 overexpression. We observed similar results at the mRNA level. 
A

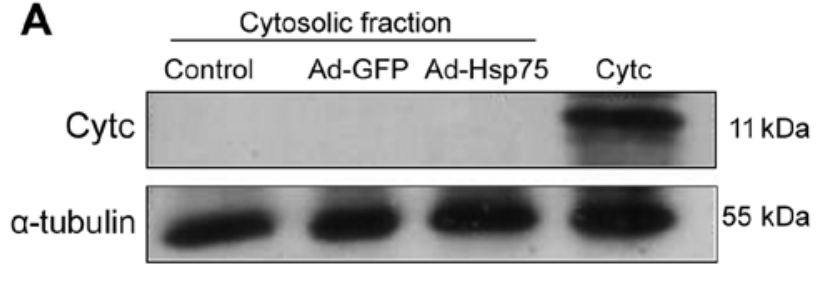

B

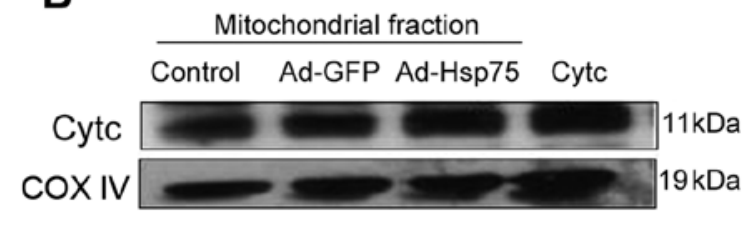

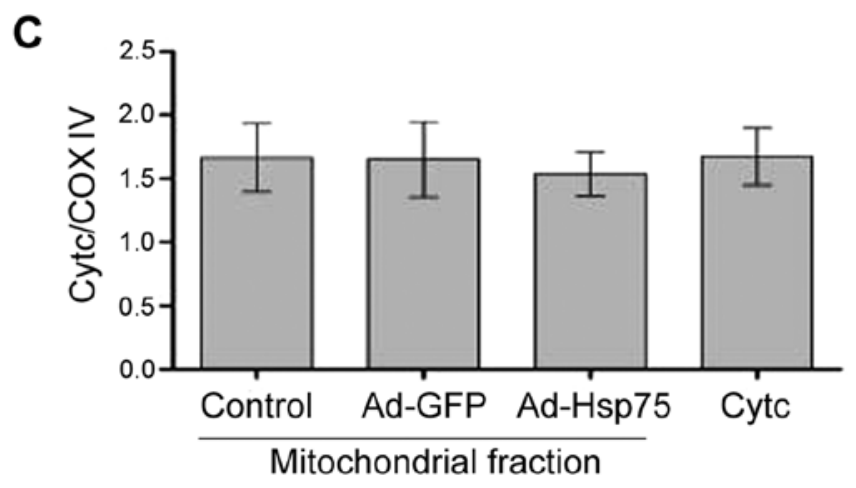

Figure 7. Lack of cytochrome $c$ (Cytc) protein expression in the cytosolic fraction. (A) The Cytc in the cytosolic fraction was analyzed by western blot analysis. $\alpha$-tubulin was used as an internal control for cytosolic protein. (B) Cytc in the mitochondria was analyzed by western blot analysis. COX IV protein served as an internal control for mitochondrial protein. (C) Quantitative analysis of Cytc protein expression in mitochondria. Data represent the means \pm SEM. Three independent experiments were performed. Ad-GFP, adenovirus green fluorescent protein; Ad-Hsp75, adenovirus heat shock protein 75.

Hsp75 overexpression prevents the soluble factor-induced release of $C y t c$. The opening of the $\mathrm{mPTP}$ ultimately results in mitochondrial membrane potential dissipation, mitochondrial swelling, rupture of the outer membrane and the release of the pro-apoptotic factor, Cytc, from the mitochondrial matrix into the cytoplasm $(13,34)$. We hypothesized that Hsp75 overexpression may reduce the soluble factor-induced release of Cytc.

The total amount of Cytc expression was measured by western blot analysis using NSCs (C17.2 cells) transfected with adenovirus. In the cytosolic fraction, no signal was observed in the NSCs (Fig. 7A), which is proof of the successful separation of the cytosolic fraction. In the mitochondrial fraction, there were no significant differences among the groups, and a strong signal was detected (Fig. 7B and C). These results indicated that the mitochondria were successfully separated, and that the total amount of Cytc protein expression was not altered following transfection with adenovirus.

To further determine whether Hsp75 overexpression reduces the soluble factor-induced release of Cytc, the release of mitochondrial Cytc into the cytosol was assessed by western blot analysis. Indeed, compared with the control group, the levels of Cytc increased significantly in the C17.2 cells that were directly exposed to $A \beta_{1-42}$. However, there was no clear difference between the NSC-MG cells (in our co-culture system; MG group) without $A \beta_{1-42}$ treatment and the control group. In addition, in the co-culture system, soluble factors significantly increased the release of Cytc in the NSC-MG cells treated with $A \beta_{1-42}[\mathrm{MG}(\mathrm{A} \beta)$ group] and in the vector-infected $\mathrm{C} 17.2$ cells (Ad-GFP group) compared to the control group, whereas cells overexpressing Hsp75 exhibited a marked decrease in the release of Cytc. These data demonstrate that Hsp75 overexpression prevents the release of Cytc induced by soluble factors (Fig. 8).
Hsp75 overexpression prevents the soluble factor-induced activation of caspase-3. It has previously been demonstrated that mitochondrial Cytc release promotes apoptotic signaling and the activation of caspase-dependent apoptotic pathways $(13,35)$. Caspase-3 activation results in DNA breakage, nuclear chromatin condensation and ultimately leads to apoptosis. In the present study, the results obtained for caspase-3 activation were similar to the above-mentioned results obtained for Cytc release (Fig. 9). Thus, Hsp75 overexpression also led to a significant attenuation of soluble factor-induced caspase- 3 activation, indicating that Hsp75 overexpression protects $\mathrm{C} 17.2$ cells against soluble factor-induced neurotoxicity by inhibiting the mitochondrial caspase-dependent apoptosis pathway.

\section{Discussion}

In the present study, we verified that C17.2 cells were nestin-positive and had relatively high transfection rates with the recombinant adenoviral vector. In addition, we found that the morphology of the $\mathrm{C} 17.2$ cells was not altered following transfection. Therefore, the use of a recombinant adenoviral vector is a suitable approach for determining whether Hsp75 provides protection against MG-derived soluble factor-induced neurotoxicity. Moreover, we demonstrated that treatment with $\mathrm{A} \beta_{1-42}$ in a co-culture system induced a time-dependent increase in the protein levels of Hsp75. Thus, MG-derived soluble factors induce an increase in the levels of Hsp75. Our data also indicate that the overproduction of Hsp75 inhibits soluble factorinduced depolarization of mitochondrial membrane potential and cell death, reducing the abundance of $\mathrm{mPTP}$, regulating CypD protein and inhibiting mitochondrial Cytc release and caspase-3 activity. In short, MG-derived soluble factors induce 
A

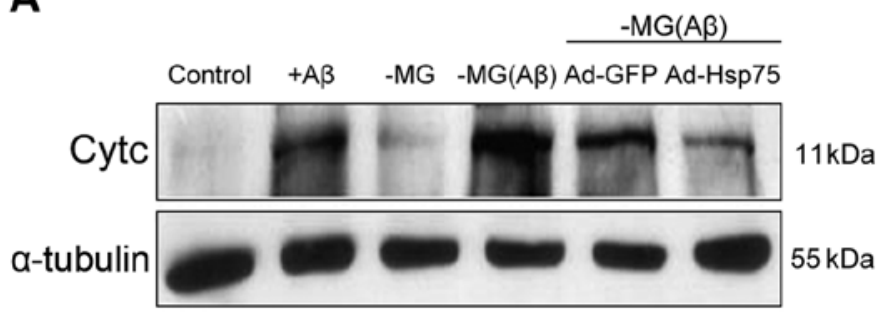

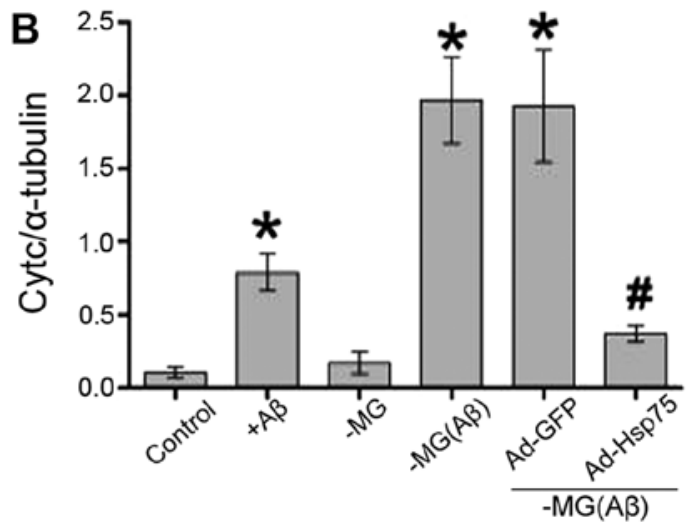

Figure 8. Determination of cytochrome $c$ (Cytc) release from mitochondria. (A) The release of Cytc into the cytosol was analyzed by western blot analysis $\alpha$-tubulin was used as an internal control for cytosolic protein. (B) Quantitative analysis of Cytc protein release. Release of the targeted protein was relative to the expression of $\alpha$-tubulin protein. Data represent the means \pm SEM. ${ }^{*} \mathrm{p}<0.05$, compared to the control group; ${ }^{*} \mathrm{p}<0.05$ compared to the neural stem cell-microglial (NSC-MG) cells treated with $\beta$-amyloid peptide $(A \beta)_{1-42}$ and the adenovirus green fluorescent protein (Ad-GFP) group. Three independent experiments were performed. Ad-Hsp75, adenovirus heat shock protein 75.

A
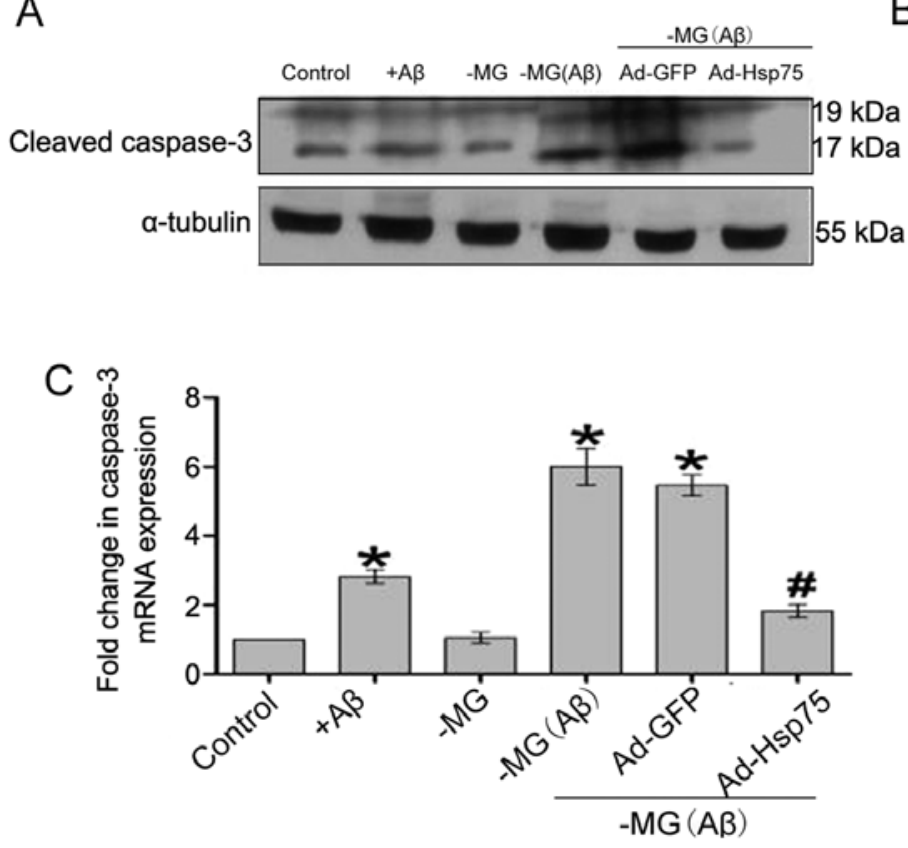
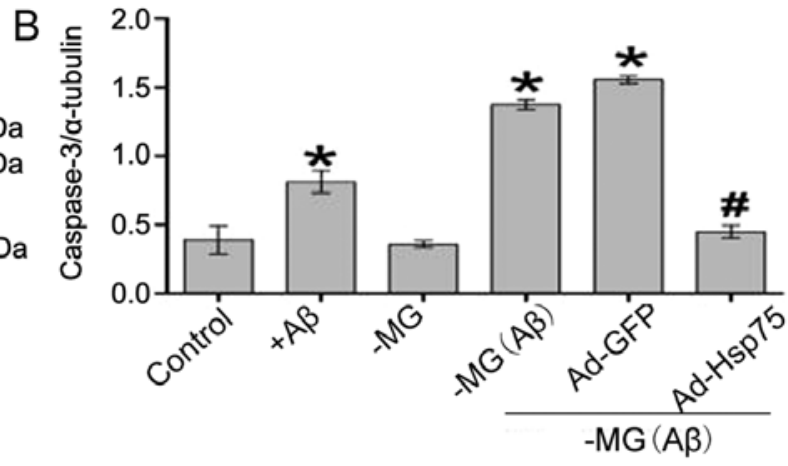

Figure 9. The level of caspase-3 was analyzed by western blot analysis and RT-qPCR. (A) Western blot analysis of caspase-3 and $\alpha$-tubulin protein expression in neural stem cells (NSCs) following treatment. (B) Quantitative analysis of caspase-3 protein expression. The expression of the targeted protein was determined relative to the expression of $\alpha$-tubulin protein. (C) RT-qPCR analysis of caspase-3 mRNA levels expressed in NSCs following treatment. The RT-qPCR experiments were performed in triplicate, and caspase-3 mRNA expression was normalized to the expression of $\beta$-actin. Data represent the means \pm SEM. ${ }^{*}$ p $<0.05$ compared to the control group; ${ }^{*} \mathrm{p}<0.05$ compared to the NSC-microglial cells (NSC-MG cells; co-culture system) treated with $\beta$-amyloid peptide $(\mathrm{A} \beta)_{1-42}$ and the adenovirus green fluorescent protein (Ad-GFP) group. Three independent experiments were performed. Ad-Hsp75, adenovirus heat shock protein 75.

mPTP opening, and concurrently, the pore opening results in the loss of mitochondrial membrane potential, the release of Cytc into the cytosol and, finally, the activation of a caspase cascade, leading to apoptosis. These results suggest that Hsp75 overexpression protects against soluble factor-induced apoptosis by blocking the consequent apoptotic cascade which is mediated by mitochondria, as shown by the inhibition of CypDdependent mPTP opening, reduced mitochondrial Cytc release and decreased caspase- 3 activity. Collectively, to the best of our knowledge, these results constitute the first evidence (relating to $\mathrm{AD}$ ) that the protective effects of Hsp75 overexpression on soluble factor-induced neurotoxicity are attributable to the role which Hsp75 plays in maintaining mitochondrial function.

Hsp75 is a heat shock protein, and like other Hsp70 members, it has been shown to be upregulated by diverse damaging stimuli: a previous study demonstrated that hypoxic injury increases Hsp75 levels in a time-dependent manner in cardiomyocytes (21). Moreover, deferoxamine, an iron chelator, has been shown to markedly decrease Hsp75 levels in a timeand dose-dependent manner in a normal human hepatocyte cell line (36). However, the changes in Hsp75 expression in NSCs following exposure to MG-derived soluble factors remain 
unclear. Based on the results of the present study, we concluded that soluble factors induce a time-dependent increase in Hsp75 levels in NSCs.

Although we verified that soluble factors induce increased levels of Hsp75, the effect of the increase in Hsp75 remains to be explored. Therefore, we constructed NSCs overexpressing human Hsp75 protein in order to determine whether the increase in Hsp75 is a protective reaction in NSCs. Previous studies have demonstrated that Hsp75 is a mitochondrial molecular chaperone and anti-apoptotic protein; Hsp75 overexpression can suppress apoptosis induced by hypoxia in cardiomyocytes (21), ischemic injury in primary astrocytes (37), glucose deprivation in PC12 cells (16) and CHL cells (38), mercury exposure in renal cells (39), arsenite exposure in lung epithelial cells (40), iron chelation in a human hepatocyte cell line (36) and $\mathrm{A} \beta_{1-42}$-induced neurotoxicity in the SH-SY5Y cell line (22). We also demonstrated in the present study that Hsp75 overexpression exerts a protective effect against MG-derived soluble factor-induced neurotoxicity in an NSC cell line (C17.2 cells).

Previous biochemical studies have indicated that diverse damaging stimuli induce mPTP formation and that MPTP formation is closely related to AD $(41,42)$. The mPTP consists of the voltage-dependent anion channel (VDAC), the adenine nucleotide translocator (ANT) and CypD. Although its exact structure remains controversial, CypD is generally accepted as a key regulatory component of the mPTP. Increasing evidence indicates that CypD contributes to the formation of MPTP and that $\mathrm{mPTP}$ formation results in severe mitochondrial membrane potential dissipation and the release of pro-apoptotic factors, which eventually leads to cell damage. In addition, it should be noted that Hsp75 is a mitochondrial molecular chaperone that maintains mitochondrial functions and is associated with the mPTP. As previously shown by Qu et al (23), Hsp75 overexpression improves cell viability and decreases apoptosis by regulating mPTP opening. Similarly, Hsp75 overexpression also protects cells against hypoxic injury by reducing $\mathrm{MPTP}$ opening (21). Therefore, we hypothesized that Hsp75 overexpression may also exert a protective effect during exposure to MG-derived soluble factors by regulating CypD-dependent mPTP opening in NSCs. In the present study, we verified that the increase in CypD expression was prevented by Hsp75 overexpression, and thus mPTP opening was decreased and the apoptotic pathways were subsequently inhibited.

In conclusion, to the best of our knowledge, our results provide the first evidence that Hsp75 is an effective protective protein against soluble factor-induced neurotoxicity and that the protective effects are related to the elimination of mitochondrial dysfunction. However, our experiments were limited to the assessment of Hsp75 overexpression and a cellular model of $\mathrm{AD}$, and therefore, further studies are required to determine whether Hsp75 overexpression provides significant protection against AD in animal models. In addition, the silencing of Hsp75 expression in vivo and in vitro needs be assessed in future studies. Finally, further investigations are warranted to examine whether soluble factors promote CypD translocation to the inner membrane to trigger mPTP opening and whether Hsp75 overexpression prevents CypD translocation. It may thus be possible to develop a novel therapeutic target for the treatment of AD based on the manipulation of Hsp75 expression.

\section{Acknowledgements}

This study was supported in part by grants from the National Natural Science Foundation of China (no. 30973162) and the Natural Science Foundation of Guangdong Province (no. S2013010015546). In addition, this study was supported in part by the Guangdong Provincial Key Laboratory of Malignant Tumor Epigenetics and Gene Regulation, the Sun Yat-Sen Memorial Hospital and Sun Yat-Sen University.

\section{References}

1. Mattson MP: Pathways towards and away from Alzheimer's disease. Nature 430: 631-639, 2004.

2. Gómez-Isla T, Hollister R, West H, Mui S, Growdon JH, Petersen RC, Parisi JE and Hyman BT: Neuronal loss correlates with but exceeds neurofibrillary tangles in Alzheimer's disease. Ann Neurol 41: 17-24, 1997.

3. Sadigh-Eteghad S, Sabermarouf B, Majdi A, Talebi M, Farhoudi M and Mahmoudi J: Amyloid-Beta: a Crucial Factor in Alzheimer's Disease. Med Princ Pract, 2014.

4. McGeer PL and McGeer EG: Targeting microglia for the treatment of Alzheimer's disease. Expert Opin Ther Targets 19: 497-506, 2014

5. Floden AM and Combs CK: Microglia demonstrate agedependent interaction with amyloid- $\beta$ fibrils. J Alzheimers Dis 25: 279-293, 2011.

6. Westerlund U, Moe MC, Varghese M, Berg-Johnsen J, Ohlsson M, Langmoen IA and Svensson M: Stem cells from the adult human brain develop into functional neurons in culture. Exp Cell Res 289: 378-383, 2003.

7. Temple S: The development of neural stem cells. Nature 414: 112-117, 2001.

8. Henn A, Lund S, Hedtjärn M, Schrattenholz A, Pörzgen P and Leist $\mathrm{M}$ : The suitability of BV2 cells as alternative model system for primary microglia cultures or for animal experiments examining brain inflammation. ALTEX 26: 83-94, 2009.

9. Lundqvist J, El Andaloussi-Lilja J, Svensson C, Gustafsson Dorfh $\mathrm{H}$ and Forsby A: Optimisation of culture conditions for differentiation of C17.2 neural stem cells to be used for in vitro toxicity tests. Toxicol In Vitro 27: 1565-1569, 2013.

10. Liu WG, Lu GQ, Li B and Chen SD: Dopaminergic neuroprotection by neurturin-expressing c17.2 neural stem cells in a rat model of Parkinson's disease. Parkinsonism Relat Disord 13: 77-88, 2007.

11. Zamzami N, Larochette N and Kroemer G: Mitochondrial permeability transition in apoptosis and necrosis. Cell Death Differ 12 (Suppl 2): 1478-1480, 2005.

12. Kim HS, Lee JH, Lee JP, Kim EM, Chang KA, Park CH, Jeong SJ, Wittendorp MC, Seo JH, Choi SH and Suh YH: Amyloid beta peptide induces cytochrome $\mathrm{C}$ release from isolated mitochondria. Neuroreport 13: 1989-1993, 2002.

13. Brustovetsky N, Brustovetsky T, Jemmerson R and Dubinsky JM: Calcium-induced cytochrome $c$ release from CNS mitochondria is associated with the permeability transition and rupture of the outer membrane. J Neurochem 80: 207-218, 2002.

14. Bhattacharyya T, Karnezis AN, Murphy SP, Hoang T, Freeman BC, Phillips B and Morimoto RI: Cloning and subcellular localization of human mitochondrial hsp70. J Biol Chem 270: 1705-1710, 1995.

15. Lee AS: The glucose-regulated proteins: stress induction and clinical applications. Trends Biochem Sci 26: 504-510, 2001.

16. Liu Y, Liu W, Song XD and Zuo J: Effect of GRP75/mthsp70/PBP74/ mortalin overexpression on intracellular ATP level, mitochondrial membrane potential and ROS accumulation following glucose deprivation in PC12 cells. Mol Cell Biochem 268: 45-51, 2005.

17. Carette J,Lehnert S and Chow TY:Implication of PBP74/mortalin/ GRP75 in the radio-adaptive response. Int J Radiat Biol 78: 183-190, 2002.

18. Massa SM, Longo FM, Zuo J, Wang S, Chen J and Sharp FR: Cloning of rat grp75, an hsp70-family member, and its expression in normal and ischemic brain. J Neurosci Res 40: 807-819, 1995.

19. Tsuchiya D, Hong S, Matsumori Y, Shiina H, Kayama T, Swanson RA, Dillman WH, Liu J, Panter SS and Weinstein PR: Overexpression of rat heat shock protein 70 is associated with reduction of early mitochondrial cytochrome $c$ release and subsequent DNA fragmentation after permanent focal ischemia. J Cereb Blood Flow Metab 23: 718-727, 2003. 
20. Rokutan K, Hirakawa T, Teshima S, Nakano Y, Miyoshi M, Kawai T, Konda E, Morinaga H, Nikawa T and Kishi K: Implications of heat shock/stress proteins for medicine and disease. J Med Invest 44: 137-147, 1998.

21. Xiang F, Huang YS, Shi XH and Zhang Q: Mitochondrial chaperone tumour necrosis factor receptor-associated protein 1 protects cardiomyocytes from hypoxic injury by regulating mitochondrial permeability transition pore opening. FEBS J 277: 1929-1938, 2010

22. Qu M, Zhou Z, Xu S, Chen C, Yu Z and Wang D: Mortalin overexpression attenuates beta-amyloid-induced neurotoxicity in SH-SY5Y cells. Brain Res 1368: 336-345, 2011.

23. Qu M, Zhou Z, Chen C, Li M, Pei L, Yang J, Wang Y, Li L, Liu C, Zhang G, et al: Inhibition of mitochondrial permeability transition pore opening is involved in the protective effects of mortalin overexpression against beta-amyloid-induced apoptosis in SH-SY5Y cells. Neurosci Res 72: 94-102, 2012.

24. Sugrue MM, Wang Y, Rideout HJ, Chalmers-Redman RM and Tatton WG: Reduced mitochondrial membrane potential and altered responsiveness of a mitochondrial membrane megachannel in p53-induced senescence. Biochem Biophys Res Commun 261: 123-130, 1999.

25. Saotome M, Katoh $H$, Satoh $H$, Nagasaka S, Yoshihara S, Terada $H$ and Hayashi H: Mitochondrial membrane potential modulates regulation of mitochondrial $\mathrm{Ca}^{2+}$ in rat ventricular myocytes. Am J Physiol Heart Circ Physiol 288: H1820-H1828, 2005.

26. Lee CS, Park SY, Ko HH, Song JH, Shin YK and Han ES Inhibition of $\mathrm{MPP}^{+}$-induced mitochondrial damage and cell death by trifluoperazine and W-7 in PC12 cells. Neurochem Int 46: 169-178, 2005.

27. Leung AW and Halestrap AP: Recent progress in elucidating the molecular mechanism of the mitochondrial permeability transition pore. Biochim Biophys Acta 1777: 946-952, 2008.

28. Halestrap AP: What is the mitochondrial permeability transition pore? J Mol Cell Cardiol 46: 821-831, 2009.

29. Gutiérrez-Aguilar M and Baines CP: Structural mechanisms of cyclophilin D-dependent control of the mitochondrial permeability transition pore. Biochim Biophys Acta: pii: S03044165(14)00386-9, 2014

30. Baines CP, Kaiser RA, Purcell NH, Blair NS, Osinska H, Hambleton MA, Brunskill EW, Sayen MR, Gottlieb RA, Dorn GW, et al: Loss of cyclophilin D reveals a critical role for mitochondrial permeability transition in cell death. Nature 434 658-662, 2005

31. Du H, Guo L, Fang F, Chen D, Sosunov AA, McKhann GM, Yan Y, Wang C, Zhang H, Molkentin JD, et al: Cyclophilin D deficiency attenuates mitochondrial and neuronal perturbation and ameliorates learning and memory in Alzheimer's disease. Nat Med 14: 1097-1105, 2008.
32. Matas J, Young NT, Bourcier-Lucas C, Ascah A, Marcil M, Deschepper CF and Burelle Y: Increased expression and intramitochondrial translocation of cyclophilin-D associates with increased vulnerability of the permeability transition pore to stress-induced opening during compensated ventricular hypertrophy. J Mol Cell Cardiol 46: 420-430, 2009.

33. Du H, Guo L, Zhang W, Rydzewska M and Yan S: Cyclophilin D deficiency improves mitochondrial function and learning/memory in aging Alzheimer disease mouse model. Neurobiol Aging 32: 398-406, 2011.

34. Kim JS, He L and Lemasters JJ: Mitochondrial permeability transition: a common pathway to necrosis and apoptosis. Biochem Biophys Res Commun 304: 463-470, 2003.

35. Yuan J, Murrell GA, Trickett A and Wang MX: Involvement of cytochrome $c$ release and caspase- 3 activation in the oxidative stress-induced apoptosis in human tendon fibroblasts. Biochim Biophys Acta 1641: 35-41, 2003.

36. Im CN, Lee JS, Zheng Y and Seo JS: Iron chelation study in a normal human hepatocyte cell line suggests that tumor necrosis factor receptor-associated protein 1 (TRAP1) regulates production of reactive oxygen species. J Cell Biochem 100: 474-486, 2007.

37. Voloboueva LA, Duan M, Ouyang Y, Emery JF, Stoy C and Giffard RG: Overexpression of mitochondrial Hsp70/Hsp75 protects astrocytes against ischemic injury in vitro. J Cereb Blood Flow Metab 28: 1009-1016, 2008.

38. Zheng DH, Zuo J, Yang ZJ, Xia BL and Zhang XN: grp75 protects cells from injuries caused by glucose deprivation. Yi Chuan Xue Bao 27: 666-671, 2000 (In Chinese)

39. Stacchiotti A, Ricci F, Rezzani R, Li Volti G, Borsani E, Lavazza A, Bianchi R and Rodella LF: Tubular stress proteins and nitric oxide synthase expression in rat kidney exposed to mercuric chloride and melatonin. J Histochem Cytochem 54: 1149-1157, 2006.

40. Lau AT, He QY and Chiu JF: A proteome analysis of the arsenite response in cultured lung cells: evidence for in vitro oxidative stress-induced apoptosis. Biochem J 382: 641-650, 2004.

41. Du H and Yan SS: Mitochondrial permeability transition pore in Alzheimer's disease: cyclophilin D and amyloid beta. Biochim Biophys Acta 1802: 198-204, 2010.

42. Rao VK, Carlson EA and Yan SS: Mitochondrial permeability transition pore is a potential drug target for neurodegeneration. Biochim Biophys Acta 1842: 1267-1272, 2014. 